\title{
EFFECT OF MAGNETIC FIELD ON THERMAL INSTABILITY OF A ROTATING RIVLIN-ERICKSEN VISCOELASTIC FLUID
}

\author{
PARDEEP KUMAR, HARI MOHAN, AND ROSHAN LAL
}

Received 22 February 2005; Revised 6 September 2005; Accepted 23 October 2005

The thermal instability of a rotating Rivlin-Ericksen viscoelastic fluid in the presence of uniform vertical magnetic field is considered. For the case of stationary convection, Rivlin-Ericksen viscoelastic fluid behaves like a Newtonian fluid. It is found that rotation has a stabilizing effect, whereas the magnetic field has both stabilizing and destabilizing effects. Graphs have been plotted by giving numerical values to the parameters, to depict the stability characteristics. The rotation and magnetic field are found to introduce oscillatory modes in the system which were nonexistent in their absence.

Copyright (c) 2006 Hindawi Publishing Corporation. All rights reserved.

\section{Introduction}

The thermal instability of a fluid layer with maintained adverse temperature gradient by heating the underside plays an important role in geophysics, interior of the Earth, oceanography, and the atmospheric physics, and so forth, and has been investigated by several authors (e.g., Bénard [1], Rayleigh [8], Jeffreys [6]) under different conditions.

A detailed account of the theoretical and experimental study of thermal instability (Bénard convection) in Newtonian fluids, under varying assumptions of hydrodynamics and hydromagnetics, has been given by Chandrasekhar [4]. The use of Boussinesq approximation has been made throughout, which states that the density may be treated as a constant in all the terms in the equations of motion except the external force term. Bhatia and Steiner [2] have considered the effect of a uniform rotation on the thermal instability of a viscoelastic (Maxwell) fluid and have found that rotation has a destabilizing influence in contrast to the stabilizing effect on Newtonian fluid. The thermal instability of a Maxwell fluid in hydromagnetics has been studied by Bhatia and Steiner [3]. They have found that the magnetic field stabilizes a viscoelastic (Maxwell) fluid just as the Newtonian fluid. Sharma [10] has studied the thermal instability of a layer of viscoelastic (Oldroydian) fluid acted on by a uniform rotation and found that rotation has destabilizing as well as stabilizing effects under certain conditions in contrast to that of a Maxwell fluid where it has a destabilizing effect. In another study, Sharma [9] has studied the stability

Hindawi Publishing Corporation

International Journal of Mathematics and Mathematical Sciences

Volume 2006, Article ID 28042, Pages 1-10

DOI 10.1155/IJMMS/2006/28042 
of a layer of an electrically conducting Oldroyd fluid [7] in the presence of a magnetic field and has found that the magnetic field has a stabilizing influence.

There are many elastico-viscous fluids that cannot be characterized by Maxwell's constitutive relations or Oldroyd's [7] constitutive relations. One such class of elasticoviscous fluids is Rivlin-Ericksen fluid. Srivastava and Singh [13] have studied the unsteady flow of a dusty elastico-viscous Rivlin-Ericksen fluid through channels of different crosssections in the presence of a time-dependent pressure gradient. In another study, Garg et al. [5] have studied the rectilinear oscillations of a sphere along its diameter in a conducting dusty Rivlin-Ericksen fluid in the presence of a uniform magnetic field. Sharma and Kumar [11] have studied the effect of rotation on thermal instability in Rivlin-Ericksen elastico-viscous fluid and found that rotation has a stabilizing effect and introduces oscillatory modes in the system. A layer of such fluid heated from below under the action of magnetic field and rotation may find applications in geophysics, interior of the Earth, oceanography, and the atmospheric physics.

Keeping in mind the importance of non-Newtonian fluids, convection in fluid layer heated from below, magnetic field, and rotation, the present paper attempts to study the effect of uniform vertical magnetic field on Rivlin-Ericksen viscoelastic fluid heated from below in the presence of a uniform rotation.

\section{Formulation of the problem and perturbation equations}

Consider an infinite, horizontal, incompressible electrically conducting Rivlin-Ericksen viscoelastic fluid layer of thickness $d$, heated from below so that the temperatures and densities at the bottom surface $z=0$ are $T_{0}$ and $\rho_{0}$ and at the upper surface $z=d$ are $T_{d}$ and $\rho_{d}$, respectively, and that a uniform temperature gradient $\beta(=|d T / d z|)$ is maintained. The gravity field $\vec{g}(0,0,-g)$, a uniform vertical magnetic field $\vec{H}(0,0, H)$, and a uniform vertical rotation $\vec{\Omega}(0,0, \Omega)$ act on the system.

The equations of motion, continuity, heat conduction, and Maxwell's equations governing the flow of Rivlin-Ericksen viscoelastic fluid in the presence of magnetic field and rotation are

$$
\begin{gathered}
\frac{\partial \vec{v}}{\partial t}+(\vec{v} \cdot \nabla) \vec{v}=-\nabla\left(\frac{p}{\rho_{0}}-\frac{1}{2}|\vec{\Omega} \times \vec{r}|^{2}\right)+\vec{g}\left(1+\frac{\delta \rho}{\rho_{0}}\right)+\left(v+v^{\prime} \frac{\partial}{\partial t}\right) \nabla^{2} \vec{v} \\
+\frac{\mu_{e}}{4 \pi \rho_{0}}(\nabla \times \vec{H}) \times \vec{H}+2(\vec{v} \times \vec{\Omega}), \\
\nabla \cdot \vec{v}=0, \\
\frac{\partial T}{\partial t}+(\vec{v} \cdot \nabla) T=\chi \nabla^{2} T, \\
\nabla \cdot \vec{H}=0, \\
\frac{\partial \vec{H}}{\partial t}=(\vec{H} \cdot \nabla) \vec{v}+\eta \nabla^{2} \vec{H},
\end{gathered}
$$

where $\vec{v}(u, v, w), p, \rho, T, v$, and $v^{\prime}$ denote the velocity, pressure, density, temperature, kinematic viscosity, and kinematic viscoelasticity, respectively, and $\vec{r}(x, y, z)$. 
The equation of state for the fluid is

$$
\rho=\rho_{0}\left[1-\alpha\left(T-T_{0}\right)\right]
$$

where $\rho_{0}, T_{0}$ are, respectively, the density and temperature of the fluid at the reference level $z=0$ and $\alpha$ is the coefficient of thermal expansion. In writing (2.1), we made use of the Boussinesq approximation, which states that the density variations are ignored in all terms in the equations of motion except the external force term. The magnetic permeability $\mu_{e}$, thermal diffusivity $\chi$, and electrical resistivity $\eta$ are all assumed to be constant.

The initial state is one in which the velocity, density, pressure, and temperature at any point in the fluid are, respectively, given by

$$
\vec{v}=(0,0,0), \quad \rho=\rho(z), \quad p=p(z), \quad T=T(z) .
$$

Let $\vec{v}(u, v, w), \delta p, \delta \rho, \theta$, and $\vec{h}\left(h_{x}, h_{y}, h_{z}\right)$ denote, respectively, the perturbations in velocity $\vec{v}$ (initially zero), pressure $p$, density $\rho$, temperature $T$, and the magnetic field $\vec{H}(0,0, H)$. The change in density $\delta \rho$, caused by the perturbation $\theta$ in temperature, is given by

$$
\rho+\delta \rho=\rho_{0}\left[1-\alpha\left(T+\theta-T_{0}\right)\right]=\rho-\alpha \rho_{0} \theta, \quad \text { i.e., } \delta \rho=-\alpha \rho_{0} \theta .
$$

Then the linearized perturbation equations are

$$
\begin{gathered}
\frac{\partial \vec{v}}{\partial t}=-\frac{1}{\rho_{0}}(\nabla \delta p)-\vec{g} \alpha \theta+\left(v+v^{\prime} \frac{\partial}{\partial t}\right) \nabla^{2} \vec{v}+\frac{\mu_{e}}{4 \pi \rho_{0}}(\nabla \times \vec{h}) \times \vec{H}+2(\vec{v} \times \vec{\Omega}), \\
\nabla \cdot \vec{v}=0, \\
\frac{\partial \theta}{\partial t}=\beta w+\chi \nabla^{2} \theta, \\
\nabla \cdot \vec{h}=0, \\
\frac{\partial \vec{h}}{\partial t}=(\vec{H} \cdot \nabla) \vec{v}+\eta \nabla^{2} \vec{h} .
\end{gathered}
$$

Within the framework of Boussinesq approximation, (2.9) become

$$
\begin{gathered}
\frac{\partial}{\partial t} \nabla^{2} w=\left(v+v^{\prime} \frac{\partial}{\partial t}\right) \nabla^{4} w+\frac{\mu_{e} H}{4 \pi \rho_{0}} \nabla^{2}\left(\frac{\partial h_{z}}{\partial z}\right)+g \alpha\left(\frac{\partial^{2} \theta}{\partial x^{2}}+\frac{\partial^{2} \theta}{\partial y^{2}}\right)-2 \Omega \frac{\partial \zeta}{\partial z}, \\
\frac{\partial \zeta}{\partial t}=\left(v+v^{\prime} \frac{\partial}{\partial t}\right) \nabla^{2} \zeta+2 \Omega \frac{\partial w}{\partial z}-\frac{\mu_{e} H}{4 \pi \rho_{0}} \frac{\partial \xi}{\partial z} \\
{\left[\frac{\partial}{\partial t}-\chi \nabla^{2}\right] \theta=\beta w} \\
{\left[\frac{\partial}{\partial t}-\eta \nabla^{2}\right] h_{z}=H \frac{\partial w}{\partial z},} \\
{\left[\frac{\partial}{\partial t}-\eta \nabla^{2}\right] \xi=H \frac{\partial \zeta}{\partial z},}
\end{gathered}
$$


4 Thermal instability of viscoelastic fluid

where $\nabla^{2}=\partial^{2} / \partial x^{2}+\partial^{2} / \partial y^{2}+\partial^{2} / \partial z^{2}$ and $\zeta=\partial v / \partial x-\partial u / \partial y ; \xi=\partial h_{y} / \partial x-\partial h_{x} / \partial y$ stand for the $z$-components of vorticity and current density, respectively.

\section{Dispersion relation}

We now analyze the disturbances into normal modes, assuming that the perturbation quantities are of the form

$$
\left[w, \theta, h_{z}, \zeta, \xi\right]=[W(z), \Theta(z), K(z), Z(z), X(z)] \exp \left(i k_{x} x+i k_{y} y+n t\right)
$$

where $k_{x}, k_{y}$ are the wave numbers along $x$ - and $y$-directions, respectively, $k=\left(k_{x}^{2}+k_{y}^{2}\right)^{1 / 2}$ is the resultant wave number, and $n$ is the growth rate which is, in general, a complex constant.

Using expression (3.1), (2.10) in nondimensional form transform to

$$
\begin{gathered}
{\left[\sigma\left(D^{2}-a^{2}\right) W+\left(\frac{g \alpha d^{2}}{v}\right) a^{2} \Theta+\frac{2 \Omega d^{3}}{v} D Z-\frac{\mu_{e} H d}{4 \pi \rho_{0} v}\left(D^{2}-a^{2}\right) D K\right]} \\
=[1+F \sigma]\left(D^{2}-a^{2}\right)^{2} W, \\
{\left[\{1+F \sigma\}\left(D^{2}-a^{2}\right)-\sigma\right] Z=-\left(\frac{2 \Omega d}{v}\right) D W-\left(\frac{\mu_{e} H d}{4 \pi \rho_{0} v}\right) D X,} \\
{\left[D^{2}-a^{2}-p_{1} \sigma\right] \Theta=-\left(\frac{\beta d^{2}}{\chi}\right) W,} \\
{\left[D^{2}-a^{2}-p_{2} \sigma\right] K=-\left(\frac{H d}{\eta}\right) D W,} \\
{\left[D^{2}-a^{2}-p_{2} \sigma\right] X=-\left(\frac{H d}{\eta}\right) D Z,}
\end{gathered}
$$

where we have introduced new coordinates $\left(x^{\prime}, y^{\prime}, z^{\prime}\right)=(x / d, y / d, z / d)$ in new units of length $d$ and $D=d / d z^{\prime}$. For convenience, the dashes are dropped hereafter. Also we have put $a=k d, \sigma=n d^{2} / v, F=v^{\prime} / d^{2} ; p_{1}=v / \chi$ is the Prandtl number and $p_{2}=v / \eta$ is the magnetic Prandtl number.

We now consider the case where both the boundaries are free as well as perfect conductors of heat, while the adjoining medium is also perfectly conducting. The case of two free boundaries is slightly artificial, except in stellar atmospheres (see Spiegel [12]) and in certain geophysical situations where it is most appropriate. However, the case of two free boundaries allows us to obtain analytical solution without affecting the essential features of the problem. The appropriate boundary conditions, with respect to which (3.2)-(3.6) must be solved, are

$$
\begin{aligned}
& W=D^{2} W=0, \quad D Z=0, \quad \Theta=0 \quad \text { at } z=0, z=1, \\
& D X=0, \quad K=0 \text {, }
\end{aligned}
$$

on a perfectly conducting boundary. 
Using the above boundary conditions, it can be shown that all the even-order derivatives of $W$ must vanish for $z=0$ and $z=1$, and hence the proper solution of $W$ characterizing the lowest mode is

$$
W=W_{0} \sin \pi z
$$

where $W_{0}$ is a constant.

Eliminating $\Theta, K, Z$, and $X$ between (3.2)-(3.6) and substituting (3.8) in the resultant equation, we obtain the dispersion relation

$$
\begin{aligned}
R_{1}= & \left(\frac{1+x}{x}\right) \frac{\left[\left\{\left(1+i F_{1} \sigma_{1} \pi^{2}\right)(1+x)+i \sigma_{1}\right\}\left\{1+x+i \sigma_{1} p_{2}\right\}+Q_{1}\right]\left[1+x+i \sigma_{1} p_{1}\right]}{\left(1+x+i \sigma_{1} p_{2}\right)} \\
& +\frac{T_{1}\left(1+x+i \sigma_{1} p_{2}\right)\left(1+x+i \sigma_{1} p_{1}\right)}{\left\{x\left[\left\{\left(1+i F_{1} \sigma_{1} \pi^{2}\right)(1+x)+i \sigma_{1}\right\}\left\{1+x+i \sigma_{1} p_{2}\right\}+Q_{1}\right]\right\}},
\end{aligned}
$$

where $R=g \alpha \beta d^{4} / v \chi, Q=\mu_{e} H^{2} d^{2} / 4 \pi \rho_{0} v \eta, T_{A}=4 \Omega^{2} d^{4} / v^{2}$ stand for the Rayleigh-number, the Chandrasekhar number, the Taylor number, respectively, and we have also put

$$
\begin{gathered}
x=\frac{a^{2}}{\pi^{2}}, \quad R_{1}=\frac{R}{\pi^{4}}, \quad i \sigma_{1}=\frac{\sigma}{\pi^{2}}, \quad F_{1}=\pi^{2} F, \\
T_{1}=\frac{T_{A}}{\pi^{4}}, \quad Q_{1}=\frac{Q}{\pi^{2}}, \quad i=\sqrt{-1} .
\end{gathered}
$$

\section{The stationary convection}

When the instability sets in as stationary convection, the marginal state will be characterized by $\sigma=0$. Putting $\sigma=0$, the dispersion relation (3.9) reduces to

$$
R_{1}=\left(\frac{1+x}{x}\right)\left[(1+x)^{2}+Q_{1}\right]+\frac{T_{1}(1+x)^{2}}{x\left[(1+x)^{2}+Q_{1}\right]},
$$

a result given by Chandrasekhar [4, equation (59), page 202].

We thus find that for the stationary convection, the viscoelasticity parameter $F$ vanishes with $\sigma$ and Rivlin-Ericksen viscoelastic fluid behaves like an ordinary Newtonian fluid.

To study the effects of rotation and magnetic field, we examine the natures of $d R_{1} / d T_{1}$ and $d R_{1} / d Q_{1}$ analytically.

Equation (4.1) yields

$$
\begin{aligned}
& \frac{d R_{1}}{d T_{1}}=\frac{(1+x)^{2}}{x\left[(1+x)^{2}+Q_{1}\right]} \\
& \frac{d R_{1}}{d Q_{1}}=\frac{(1+x)}{x}-\frac{T_{1}(1+x)^{2}}{x\left[(1+x)^{2}+Q_{1}\right]^{2}} .
\end{aligned}
$$

It is evident from (4.2) that for a stationary convection, $d R_{1} / d T_{1}$ is always positive, thus, the rotation has a stabilizing effect on the system. It is also clear from (4.3) that for 
6 Thermal instability of viscoelastic fluid

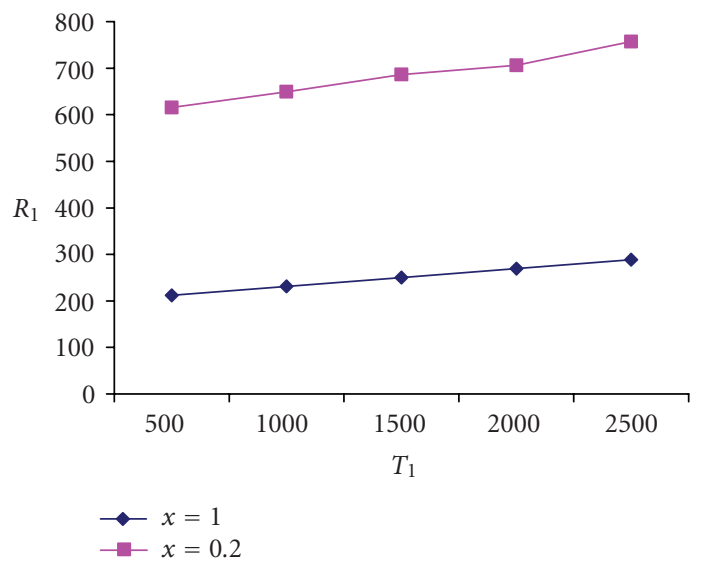

Figure 4.1. The variation of $R_{1}$ with $T_{1}$ for fixed values of $Q_{1}=100$ and $x=0.2,1$.

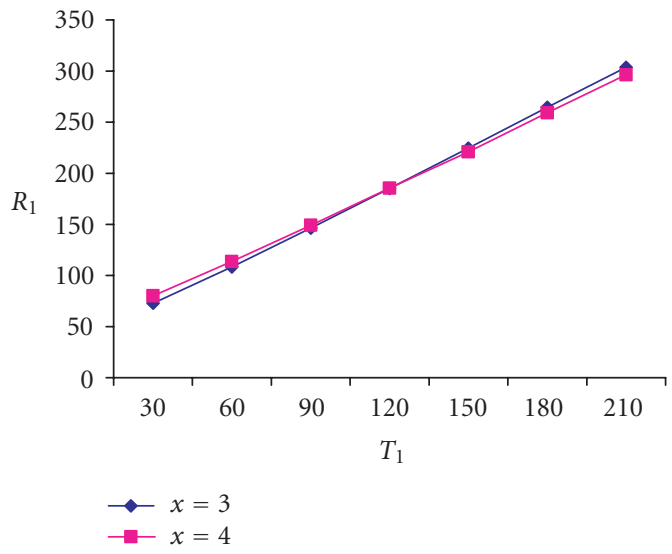

Figure 4.2. The variation of $R_{1}$ with $Q_{1}$ for fixed values of $T_{1}=100$ and $x=3,4$.

a stationary convection, $d R_{1} / d Q_{1}$ may be positive as well as negative, thus, the magnetic field has both stabilizing and destabilizing effects on the system.

The dispersion relation (4.1) is also analyzed numerically. In Figure 4.1, $R_{1}$ is plotted against $T_{1}$, for fixed value of $Q_{1}=100$ and wave numbers $x=0.2,1$. The Rayleigh number $R_{1}$ increases with increase in rotation parameter $T_{1}$ showing its stabilizing effect on the system. Figure 4.2 shows the variation of $R_{1}$ with respect to $Q_{1}$, for fixed value of $T_{1}=100$ and wave numbers $x=3,4$. It clearly depicts both the stabilizing and destabilizing effects of the magnetic field on the system.

\section{Stability of the system and oscillatory modes}

Multiplying (3.2) by $W^{*}$, the complex conjugate of $W$, integrating the resulting equation over the range of $z$ and using (3.3)-(3.6), together with the boundary conditions (3.7), 
we obtain

$$
\begin{aligned}
-\sigma I_{1} & +\frac{g \alpha \chi a^{2}}{v \beta}\left(I_{2}+p_{1} \sigma^{*} I_{3}\right)-d^{2}\left(1+F \sigma^{*}\right) I_{4}-d^{2} \sigma^{*} I_{5} \\
- & \frac{\mu_{e} d^{2} \eta}{4 \pi \rho_{0} v}\left(I_{6}+p_{2} \sigma I_{7}\right)-\frac{\mu_{e} \eta}{4 \pi \rho_{0} v}\left(I_{8}+p_{2} \sigma^{*} I_{9}\right)=(1+F \sigma) I_{10}
\end{aligned}
$$

where

$$
\begin{aligned}
& I_{1}=\int_{0}^{1}\left(|D W|^{2}+a^{2}|W|^{2}\right) d z, \quad I_{2}=\int_{0}^{1}\left(|D \Theta|^{2}+a^{2}|\Theta|^{2}\right) d z, \\
& I_{3}=\int_{0}^{1}\left(|\Theta|^{2}\right) d z, \quad I_{4}=\int_{0}^{1}\left(|D Z|^{2}+a^{2}|Z|^{2}\right) d z, \\
& I_{5}=\int_{0}^{1}\left(|Z|^{2}\right) d z, \quad I_{6}=\int_{0}^{1}\left(|D X|^{2}+a^{2}|X|^{2}\right) d z, \\
& I_{7}=\int_{0}^{1}\left(|X|^{2}\right) d z, \quad I_{8}=\int_{0}^{1}\left(\left|D^{2} K\right|^{2}+2 a^{2}|D K|^{2}+a^{4}|K|^{2}\right) d z, \\
& I_{9}=\int_{0}^{1}\left(|D K|^{2}+a^{2}|K|^{2}\right) d z, \quad I_{10}=\int_{0}^{1}\left(\left|D^{2} W\right|^{2}+2 a^{2}|D W|^{2}+a^{4}|W|^{2}\right) d z,
\end{aligned}
$$

and $\sigma^{*}$ is the complex conjugate of $\sigma$. The integrals $I_{1}, \ldots, I_{10}$ are all positive definite. Putting $\sigma=\sigma_{r}+i \sigma_{i}$, where $\sigma_{r}, \sigma_{i}$ are real and equating the real and imaginary parts of (5.1), we obtain

$$
\begin{gathered}
\sigma_{r}\left[-I_{1}+\frac{g \alpha \chi a^{2}}{v \beta} p_{1} I_{3}-d^{2} F I_{4}-d^{2} I_{5}-\frac{\mu_{e} d^{2} \eta}{4 \pi \rho_{0} v} p_{2} I_{7}-\frac{\mu_{e} \eta}{4 \pi \rho_{0} v} p_{2} I_{9}-F I_{10}\right] \\
=-\frac{g \alpha \chi a^{2}}{v \beta} I_{2}+d^{2} I_{4}+\frac{\mu_{e} d^{2} \eta}{4 \pi \rho_{0} v} I_{6}+\frac{\mu_{e} \eta}{4 \pi \rho_{0} v} I_{8}+I_{10} \\
\sigma_{i}\left[I_{1}+\frac{g \alpha \chi a^{2}}{v \beta} p_{1} I_{3}-d^{2} F I_{4}-d^{2} I_{5}+\frac{\mu_{e} d^{2} \eta}{4 \pi \rho_{0} v} p_{2} I_{7}-\frac{\mu_{e} \eta}{4 \pi \rho_{0} v} p_{2} I_{9}+F I_{10}\right]=0 .
\end{gathered}
$$

\section{Discussion}

From (5.4), it is clear that $\sigma_{i}$ is zero when the quantity multiplying it is not zero and arbitrary when this quantity is zero.

If $\sigma_{i} \neq 0$, then (5.4) gives

$$
\frac{g \alpha \chi a^{2}}{v \beta} p_{1} I_{3}-d^{2} F I_{4}-d^{2} I_{5}-\frac{\mu_{e} \eta}{4 \pi \rho_{0} v} p_{2} I_{9}=-I_{1}-\frac{\mu_{e} d^{2} \eta}{4 \pi \rho_{0} v} p_{2} I_{7}-F I_{10} .
$$

Substituting in (5.3), we have

$$
I_{10}+\frac{\mu_{e} \eta}{4 \pi \rho_{0} v} I_{8}+\frac{\mu_{e} d^{2} \eta}{4 \pi \rho_{0} v} I_{6}+d^{2} I_{4}+2 \sigma_{r}\left[I_{1}+\frac{\mu_{e} d^{2} \eta}{4 \pi \rho_{0} v} p_{2} I_{7}+F I_{10}\right]=\frac{g \alpha \chi a^{2}}{v \beta} I_{2} .
$$


8 Thermal instability of viscoelastic fluid

Equation (6.2) on using Rayleigh-Ritz inequality gives

$$
\begin{aligned}
& \frac{\left(\pi^{2}+a^{2}\right)^{3}}{a^{2}} \int_{0}^{1}|W|^{2} d z+\frac{\left(\pi^{2}+a^{2}\right)}{a^{2}} \\
& \quad \times\left\{\frac{\mu_{e} \eta}{4 \pi \rho_{0} v} I_{8}+\frac{\mu_{e} d^{2} \eta}{4 \pi \rho_{0} v} I_{6}+d^{2} I_{4}+2 \sigma_{r}\left[I_{1}+\frac{\mu_{e} d^{2} \eta}{4 \pi \rho_{0} v} p_{2} I_{7}+F I_{10}\right]\right\} \\
& \quad \leq \frac{g \alpha \chi}{\nu \beta} \int_{0}^{1}|W|^{2} d z .
\end{aligned}
$$

Therefore, it follows from (6.3) that

$$
\begin{aligned}
& {\left[\frac{27 \pi^{4}}{4}-\frac{g \alpha \chi}{\nu \beta}\right] \int_{0}^{1}|W|^{2} d z+\frac{\left(\pi^{2}+a^{2}\right)}{a^{2}}} \\
& \quad \times\left\{\frac{\mu_{e} \eta}{4 \pi \rho_{0} v} I_{8}+\frac{\mu_{e} d^{2} \eta}{4 \pi \rho_{0} v} I_{6}+d^{2} I_{4}+2 \sigma_{r}\left[I_{1}+\frac{\mu_{e} d^{2} \eta}{4 \pi \rho_{0} v} p_{2} I_{7}+F I_{10}\right]\right\} \leq 0,
\end{aligned}
$$

since minimum value of $\left(\pi^{2}+a^{2}\right)^{3} / a^{2}$ with respect to $a^{2}$ is $27 \pi^{4} / 4$.

Now, let $\sigma_{r} \geq 0$, we necessarily have from (6.4) that

$$
\frac{g \alpha \chi}{\nu \beta}>\frac{27 \pi^{4}}{4}
$$

Hence, if

$$
\frac{g \alpha \chi}{\nu \beta} \leq \frac{27 \pi^{4}}{4}
$$

then $\sigma_{r}<0$. Therefore, the system is stable.

Therefore, under condition (6.6), the system is stable and under condition (6.5) the system becomes unstable.

In the absence of rotation and magnetic field, (5.4) reduces to

$$
\sigma_{i}\left[I_{1}+\frac{g \alpha \chi a^{2}}{v \beta} p_{1} I_{3}+F I_{10}\right]=0
$$

and the terms in brackets are positive definite. Thus, $\sigma_{i}=0$, which means that oscillatory modes are not allowed and the principle of exchange of stabilities is satisfied for RivlinEricksen viscoelastic fluid heated from below.

\section{Acknowledgment}

The authors are thankful to the referee for useful technical comments and valuable suggestions, which led to a significant improvement of the paper. 


\section{Nomenclature}

$\begin{array}{ll}d & \text { Depth of layer } \\ T & \text { Temperature } \\ g & \text { Acceleration due to gravity } \\ \vec{g} & \text { Gravity field } \\ \vec{H}(0,0, H) & \text { Uniform vertical magnetic field } \\ \vec{\Omega}(0,0, \Omega) & \text { Uniform vertical rotation field } \\ \vec{v} & \text { Filter velocity } \\ p & \text { Fluid pressure } \\ \vec{r}(x, y, z) & \text { Space coordinates } \\ \delta p & \text { Perturbation in pressure } \\ \vec{h}\left(h_{x}, h_{y}, h_{z}\right) & \text { Perturbation in magnetic filed } \\ k_{x}, k_{y} & \text { Wave numbers in } x \text { - and } y \text {-directions } \\ k & \text { Resultant wave number } \\ n & \text { Growth rate } \\ p_{1} & \text { Prandtl number } \\ p_{2} & \text { Magnetic Prandtl number } \\ R & \text { Rayleigh number } \\ Q & \text { Chandrasekhar number } \\ a & \text { Dimensionless wave number } \\ F & \text { Dimensionless kinematic viscoelasticity }\end{array}$

Greek letters

$\mu$

$\mu^{\prime}$

$\rho$

$\beta$

$\nu$

$v^{\prime}$

$\mu_{e}$

$\alpha$

$\chi$

$\eta$

$\delta \rho$

$\theta$

$\varsigma$

$\xi$
Fluid viscosity

Fluid viscoelasticity

Density

Uniform temperature gradient

Kinematic viscosity

Kinematic viscoelasticity

Magnetic permeability

Coefficient of thermal expansion

Thermal diffusivity

Electrical resistivity

Perturbation in density

Perturbation in temperature

$z$-component of vorticity

$z$-component of current density

\section{References}

[1] H. Bénard, Les tourbillons cellulaires dans une nappe liquide, Revue Genérale des Sciences Pures et Appliquées 11 (1900), 1261-1271, 1309-1328.

[2] P. K. Bhatia and J. M. Steiner, Convective instability in a rotating viscoelastic fluid layer, Zeitschrift für Angewandte Mathematik und Mechanik 52 (1972), 321-327. 
[3] _ Thermal instability in a viscoelastic fluid layer in hydromagnetics, Journal of Mathematical Analysis and Applications 41 (1973), no. 2, 271-283.

[4] S. Chandrasekhar, Hydrodynamic and Hydromagnetic Stability, Dover, New York, 1981.

[5] A. Garg, R. K. Srivastava, and K. K. Singh, Drag on sphere oscillating in conducting dusty RivlinErickson elastico-viscous liquid, Proceedings of the National Academy of Sciences. India A64 (1994), no. 3, 355-363.

[6] H. Jeffreys, The stability of a fluid layer heated from below, Philosophical Magazine 2 (1926), 833-844.

[7] J. G. Oldroyd, Non-Newtonian effects in steady motion of some idealized elastico-viscous liquids, Proceedings of the Royal Society of London A245 (1958), 278-297.

[8] L. Rayleigh, On convective currents in a horizontal layer of fluid when the higher temparture is on the under side, Philosophical Magazine 32 (1916), 529-546.

[9] R. C. Sharma, Thermal instability in a viscoelastic fluid in hydromagnetics, Acta Physica Hungarica 38 (1975), 293-298.

[10] Ef_ect of rotation on thermal instability of a viscoelastic fluid, Acta Physica Hungarica 40 (1976), 11-17.

[11] R. C. Sharma and P. Kumar, Effect of rotation on thermal instability in Rivlin-Ericksen elasticoviscous fluid, Zeitschrift für Naturforschung 51a (1996), 821-824.

[12] E. A. Spiegel, Convective instability in a compressible atmosphere, The Astrophysical Journal 141 (1965), 1068.

[13] R. K. Srivastava and K. K. Singh, Drag on a sphere oscillating in a conducting dusty viscous fluid in presence of uniform magnetic filed, Bulletin of the Calcutta Mathematical Society 80 (1988), 286-292.

Pardeep Kumar: Department of Mathematics, International Centre for Distance Education and Open Learning (ICDEOL), Himachal Pradesh University, Shimla-171005, India E-mail address: drpardeep@sancharnet.in

Hari Mohan: Department of Mathematics, International Centre for Distance Education and Open Learning (ICDEOL), Himachal Pradesh University, Shimla-171005, India E-mail address: hm_math_hpu@redffmail.com

Roshan Lal: Department of Mathematics, International Centre for Distance Education and Open Learning (ICDEOL), Himachal Pradesh University, Shimla-171005, India 


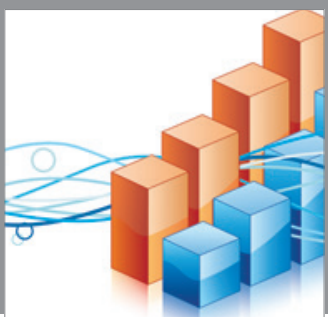

Advances in

Operations Research

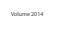

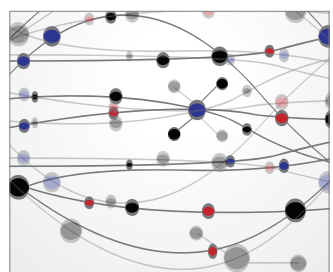

\section{The Scientific} World Journal
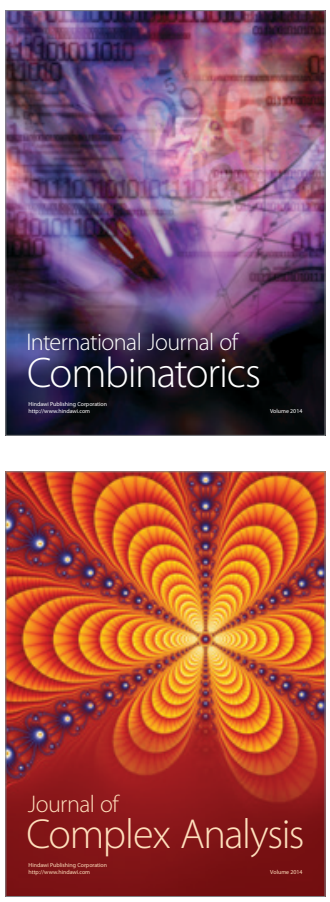

International Journal of

Mathematics and

Mathematical

Sciences
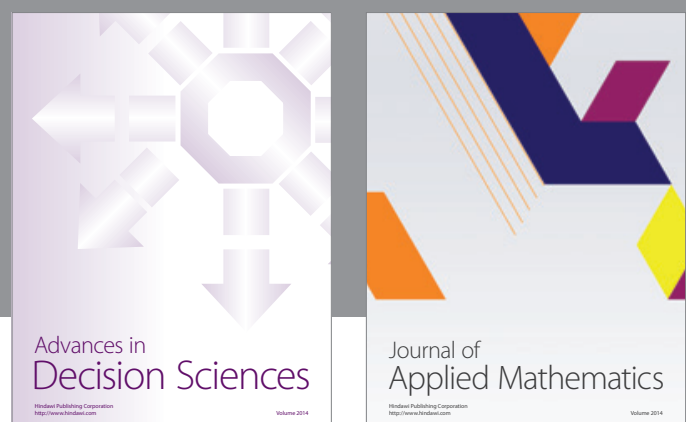

Journal of

Applied Mathematics
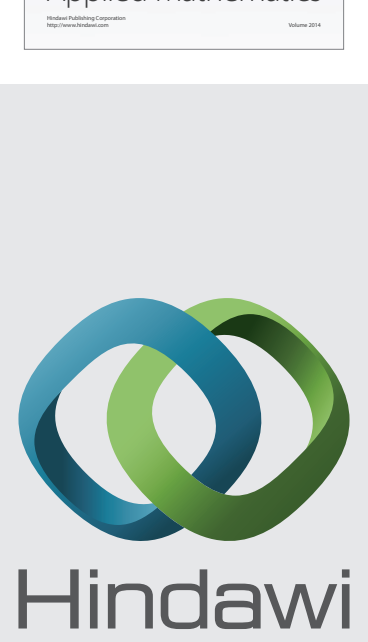

Submit your manuscripts at http://www.hindawi.com
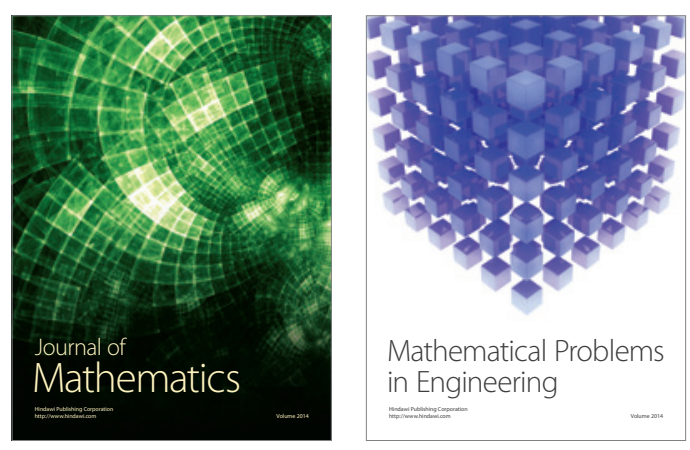

Mathematical Problems in Engineering
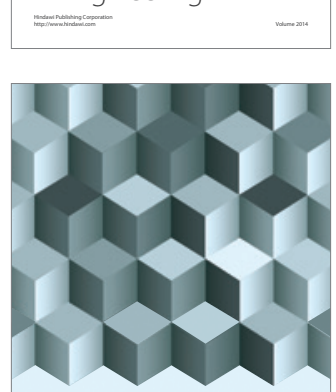

Journal of

Function Spaces
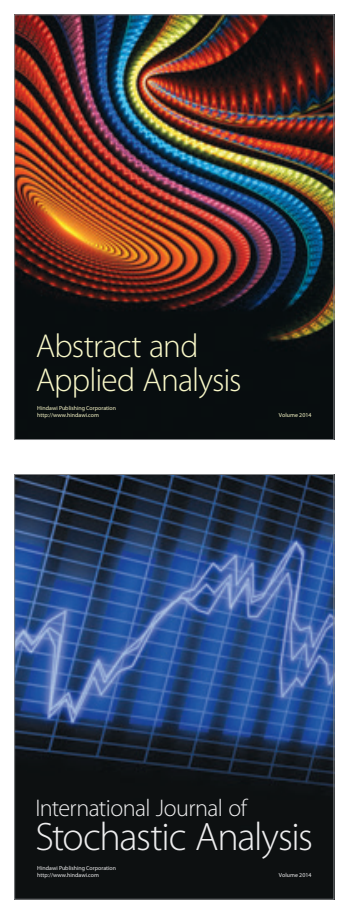

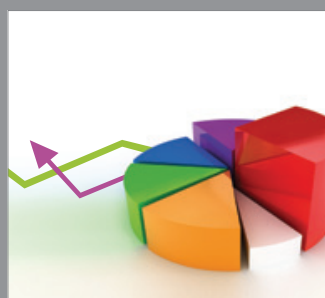

ournal of

Probability and Statistics

Promensencen
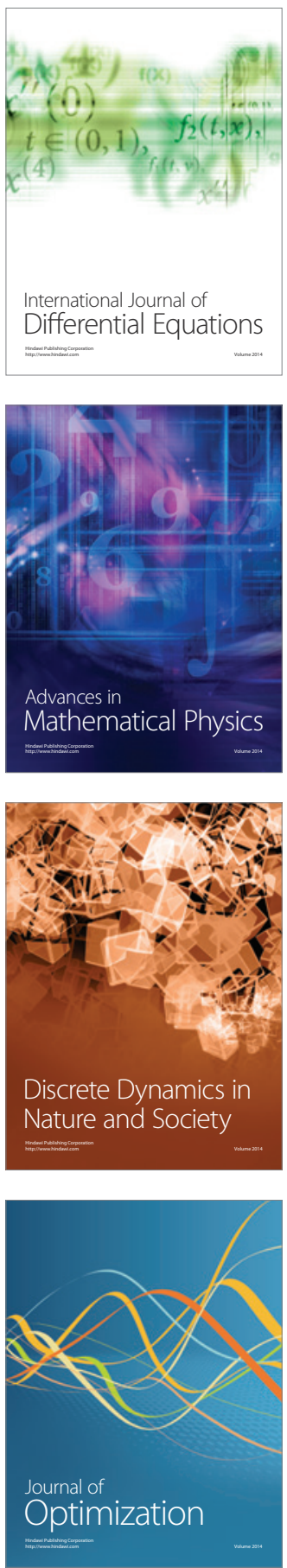